\title{
Molecular allergy diagnostics using multiplex assays: methodological and practical considerations for use in research and clinical routine
}

\section{Part 21 of the Series Molecular Allergology}

\author{
Thilo Jakob ${ }^{1}$, Peter Forstenlechner ${ }^{2}$, Paolo Matricardi ${ }^{3}$, Jörg Kleine-Tebbe ${ }^{4}$ \\ 'Department of Dermatology and Allergology, University Medical Center Gießen \& Marburg, Justus Liebig \\ University Gießen, Gießen, Germany; ${ }^{2}$ Thermo Fisher Scientific Phadia Austria GmbH, Vienna, Austria; ${ }^{3}$ Department \\ of Pediatric Pneumonology and Immunology, Charité University Hospital, Berlin, Germany; ${ }^{4}$ Westend Allergy and \\ Asthma Center, Hanf, Ackermann-Simon, and Kleine-Tebbe Specialist Allergy Practice, Berlin, Germany
}

\section{Key words \\ $\lg \mathrm{E}$ - Allergens - \\ Component- \\ based diag- \\ nostics - Multi- \\ plex - In vitro \\ test}

\section{Abstract}

The availability of single allergens and their use in microarray technology enables the simultaneous determination of specific IgE (sIgE) to a multitude of different allergens $(>100)$ in a multiplex procedure requiring only minute amounts of serum. This allows extensive individual sensitization profiles to be determined from a single analysis. Combined with a patient's medical history, these profiles simplify identification of cross-reactivity; permit a more accurate estimation of the risk of severe reactions; and enable the indication for specific immunotherapy to be more precisely established, particularly in cases of polysensitization.

Strictly speaking, a multiplex assay is not a single test, but instead more than 100 simultaneous tests. This places considerable demands on the production, quality assurance, and interpretation of data. The following chapter describes the multiplex test systems currently available and discusses their characteristics. Performance data are presented and the sIgE values obtained from multiplex and singleplex assays are compared. Finally, the advantages and limitations of molecular allergy diagnostics using multiplex assays in clinical routine are discussed, and innovative possibilities for clinical research are described.

The multiplex diagnostic tests available for clinical routine have now become well established. The interpretation of test results is demanding, particularly since all individual results need to be checked for their plausibility and clinical relevance on the basis of previous history (patient history, clinical symptoms, challenge test results). There is still room
Abbrevations
CV Coefficient of variation
DNA Deoxyribonucleic acid
EDTA Ethylenediaminetetraacetic acid
IACV Intra-assay coefficient of variation
IECV Inter-assay coefficient of variation
$\lg \mathrm{A} \quad$ Immunoglobulin $\mathrm{A}$
IgE Immunoglobulin $\mathrm{E}$
IgG Immunoglobulin $\mathrm{G}$
ISAC Immuno Solid-phase Allergen Chip
LoD Limit of detection
LoQ Limit of quantification
LTP Lipid transfer protein
RAST Radioallergosorbent test
slgE Specific immunoglobulin E
SIT Specific immunotherapy 
for improvement in certain areas, for example with respect to the overall test sensitivity of the method, as well as the availability and quality of particular allergens. The current test systems are just the beginning of a continuous development that will influence and most likely change clinical allergology in the coming years.

Cite this as: Jakob T, Forstenlechner P, Matricardi $\mathrm{P}$, Kleine-Tebbe J. Molecular allergy diagnostics using multiplex assays: methodological and practical considerations for use in research and clinical routine. Part 21 of the Series Molecular Allergology. Allergo J Int 2015;24:320-32

DOI: 10.1007/s40629-015-0056-2

\section{Introduction}

Since Charles Blackley carried out the first in vivo test with pollen on his own skin in 1880 [1], the diagnosis of type I hypersensitivity has been performed using extract preparations. Almost 90 years later, shortly after the discovery of immunoglobulin $\mathrm{E}$ (IgE), the radioallergosorbent test (RAST) was established. This test allowed for the first time the detection of circulating specific $\operatorname{IgE}(\mathrm{s} \operatorname{IgE}$ ) antibodies in vitro, using radio-labeled anti-IgE antibodies $[2,3,4]$. IgE binding to allergen extracts coupled to a solid phase (paper discs) was measured. The successful sequencing of the DNA of the major birch pollen allergen Bet $\mathrm{v} 1$ kickstarted the era of molecular allergy diagnostics [5]. Recombinant or purified (glyco-)proteins since then enabled the measurement of sIgE to defined single allergens - initially in singleplex and, since 2001, also in multiplex assays.

Multiplex assays in allergy diagnostics refer to the simultaneous determination of sIgE to different allergens or allergen extracts in a single test run. This approach has already been used in the past in the form of strip tests for allergy screening (e.g., Allergodip ${ }^{\varpi}$, Euroline, Polycheck ${ }^{\circledast}$ ), in order to obtain as much information as possible on the sensitization status of an allergic patient in a single test.

These strip tests are based on the "dotblot" principle, in which:

- multiple dot-shaped or

- strip-shaped

allergen-containing membranes serve as the solid phase. These tests enable simultaneous semiquantitative measurement of sIgE to different allergen sources; they do not, however, enable elucidation of the sensitization pattern on a molecular level, since extracts are usually used.

Only with the progress made in molecular allergology and chip-based microarray technology could multiplex assays be developed in which a patient's sIgE profile can be analyzed in detail at the level of individual molecules. To accomplish this, minute quantities (picogram range) of different allergens are coupled to a solid phase before these protein arrays (allergen chips) are used for simultaneous determination of allergen-specific IgE [6]. In contrast to single tests (singleplex assays) (for review see [7]) and extract-based diagnostics, allergen chips enable elucidation of an extensive sensitization profile at the individual-molecule level in a single measurement. This enables a differentiated analysis of the individual IgE repertoire and reveals a patient's current sensitization status.

The present article first introduces the multiplex diagnostic procedure. It then goes on to discuss the advantages and limitations of this new technology for allergy diagnostics in clinical routine and in the research environment.

\section{Molecular allergy diagnostics using multiplex assays}

Whereas singleplex assays for molecular allergy diagnostics are already used by and available from many manufacturers of diagnostic tools, there are currently only few companies that offer multiplex assays for molecular allergy diagnostics.

Of these test systems, one has established itself as the gold standard in multiplex-assay molecular allergy diagnostics. This system is based on the Immuno Solid-phase Allergen Chip (ISAC), which has been available since 2001. The ISAC was initially developed and manufactured by VBC Genomics in Vienna; since 2009, it has been further developed,

\section{Definitions}

Allergen (also single allergen Molecule with the ability to bind slgE or or allergen component)

Allergen source trigger slgE production

Organism that expresses
les (e.g., cat, grass pollen)

ISAC Immuno Solid-phase Allergen Chip, multiplex tool for the determination of slgE using microarray

Microarray

Term used for molecular biological test methods that allow parallel testing of multiple analytes (also known as bio- or allergen chip)

Multiplex assay Simultaneous testing of multiple analytes in a single assay (e.g., using microarray)

Singleplex assay Testing of a single analyte in a single assay

Diagnostic sensitivity The probability that a test yields a positive result in an affected individual

Diagnostic specificity The probability that a test yields a negative result in a healthy individual

Coefficient of variation Measure of relative scattering 
manufactured, and marketed by Phadia, Thermo Fisher Scientific, Uppsala, Sweden. Under the product name ImmunoCAP ${ }^{\circledR}$ ISAC 112 , the current version of this allergen chip enables determination of sIgE to 112 different single molecules from 51 different plant and animal allergen sources.

In addition, test systems exist that couple „classic“ allergen extracts onto chips for microarray assay, or combine a range of defined single allergens with extracts. One of these is a test system only recently CE certified for extract- and component-based diagnostics (ADAM ${ }^{\mathrm{Ts}}$, Microtest Diagnostics Ltd, London, UK). This fully automated test system can semiquantitatively determine sIgE to common aero- and food allergens within $4 \mathrm{~h}$. The test principle is based on a protein microarray currently featuring 22 allergen extracts, three recombinant proteins ( $\mathrm{rBet} \mathrm{v}$ 1 , rAra $\mathrm{h} 2$, and rCor a 1), and one purified single allergen (nGal d 1). Since virtually no technical or clinical data on the evaluation of the system are available to date, it is not possible at present to make any statement on test performance.

Another multiplex test system is currently being developed by Abionic. This system is also based on a fully automated microarray assay and enables measurement of sIgE reactivity to common single allergens in different screening panels: e.g., a screening panel with the food and inhalant allergens Gal d 1, Bos d 5, Ara h 2, Bet v 1, Bet v 2, Phl p 1, Phl p 5, Der p 1, Can f 1, and Fel d 1. The system is conceived as a "point of care“ instrument, uses capillary blood, and - according to the manufacturer's data - enables the determination of sIgE within $20 \mathrm{~min}$. There are currently no study data available on this system.

\section{Immuno Solid-phase Allergen Chip (ISAC) The test procedure}

The ImmunoCAP ${ }^{\circledast}$ ISAC 112 , a solid-phase immunoassay, comprises a polymer-coated slide with four fields, the protein microarrays (i.e., allergen chips). One array is used per patient sample, such that four different sera can be tested with each slide. The allergens (in the picogram range) are applied in triplicates, thus enabling multiple measurements, and covalently bound to the polymer layer (Fig. 1). The allergen components immobilized in this way bind all allergen-specific antibodies (e.g., IgE, IgG, IgA) in the patient sample. Once the nonspecific antibodies have been washed away, a fluorescently labeled anti-human IgE antibody is added to promote complex formation. Following incubation, unbound antibodies of other isotypes (IgG, IgA, etc.) and excess unbound fluorescently labeled anti-human IgE antibodies are removed by washing. Finally, fluorescence is measured using a microarray scanner. The higher the signal, the more sIgE is present in the sample. The test results are analyzed with PC-based software and the concentration of sIgE in the sample is calculated providing ISAC standard units (ISU-E). The manufacturer has adjusted the calibration curve to approximately match the units in the ImmunoCAP singleplex method $\left(\mathrm{kU}_{\mathrm{A}} / \mathrm{l}\right)$. The latter are derived heterologously from a total-IgE standard curve, whereas ISU-E are based on calibration using the ImmunoCAP singleplex system (Phadia 250).

Measurement values are reported not only quantitatively, but also semiquantitatively, divided into four different categories:

1. values $<0.3$ ISU-E are defined as negative

2. values between 0.3 and 1.0 ISU-E as low-level positive

3. values between 1.0 and 15.0 ISU-E as moderately high

4. values $\geq 15.0$ ISU-E as very high

Thus, test results comprise the actual measurement, plus a color-coded bar chart representation from which the approximate value of the measurement and the evaluation category can be red.

The ISAC 112 is primarily defined as a semiquantitative method, since, in the manufacturer's opinion

— the miniaturization of the assay design

- the shape of the calibration curve

- the degree of scattering

- potential divergent values due to competitive inhibition by competing allergen-specific antibodies of other classes (see below)

preclude reliable measurement of the „true“ quantitative concentrations of allergen-specific $\operatorname{IgE}$ antibodies.

\section{Test performance}

Extensive test performance data were collected for ISAC 112 by the manufacturer in 2011 [8]. These data pertain to the following parameters:

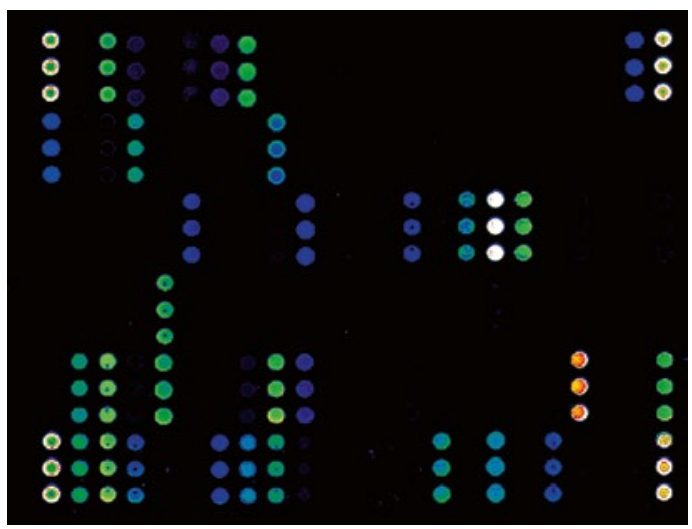

Fig. 1: Example of ISAC 112 microarray analysis with triplicate measurements of slgE signals 
- precision (reproducibility depending on signal strength)

- intra-assay coefficients of variation (IACV), interassay coefficients of variation (IECV)

_linearity (measurement response using diluted samples)

_limit of detection (LoD) and limit of quantitation (LoQ)

- matrix effects

_ total-IgE interference

-parallel comparison with singleplex tests (ImmunoCAP)

Data on precision, linearity, and LoD, as well as on factors possibly causing interference in the assay are discussed in the following sections.

Intra- and inter-assay variance: Data on precision were collected using sera from four multisensitized patients. The samples were measured in triplicate a total of 17 times over a 4 -week period. This approach generated data on intra- and inter-assay variance for 105 of 112 allergens. According to the manufacturer, the average coefficient of variation $(\mathrm{CV})$ for all allergens tested in intra- and inter-assay comparisons is below $20 \%$. However, it should be noted that the $\mathrm{CV}$ values vary depending on the test system's measurement range (0.3-1.0 ISU-E vs. 1.0-15 ISU-E vs. > 15 ISU-E), with higher values reported in the lowest measurement range (Fig. 2, Tab. 1, Tab. 2).

Linearity and limit of detection (LoD): Investigations of linearity were performed using serial $1: 2$ dilutions on sera with high sIgE values (> 5 ISU-E) to the respective allergen. In this manner, linearity curves and coefficients of determination $\left(\mathrm{R}^{2}\right)$ were calculated for 81 of the 112 allergens, which confirmed the linearity between measurement values and orders of dilution in wide ranges (Fig. 3, Tab. 3).

The LoD, defined as the lowest sIgE concentration that can be reliably determined, was determined for eight representative allergens (Ara h 1, Bet v 1, Der p 1, Equ c 1, Fel d 1, Gad c 1, Gal d 1, and Phl p 5) according to the global consensus on the standardization of healthcare technology guidelines (NCCLS-EP17-A). The LoD was between 0.05 and 0.28 ISU-E for the individual allergens. Based on these results, and considering the identical test conditions and known CV values in the lowest measurement range, an LoD of $<0.3$ ISU-E was assumed for all 112 allergens. However, according to the manufacturer, sIgE concentrations $<1 \mathrm{kU}_{\mathrm{A}} / \mathrm{l}$ are not reliably detectable by the ISAC 112 system. Therefore, the overall assay sensitivity (LoD, LoQ) of ISAC 112 is to be considered lower than that of the ImmunoCAP (singleplex) method.

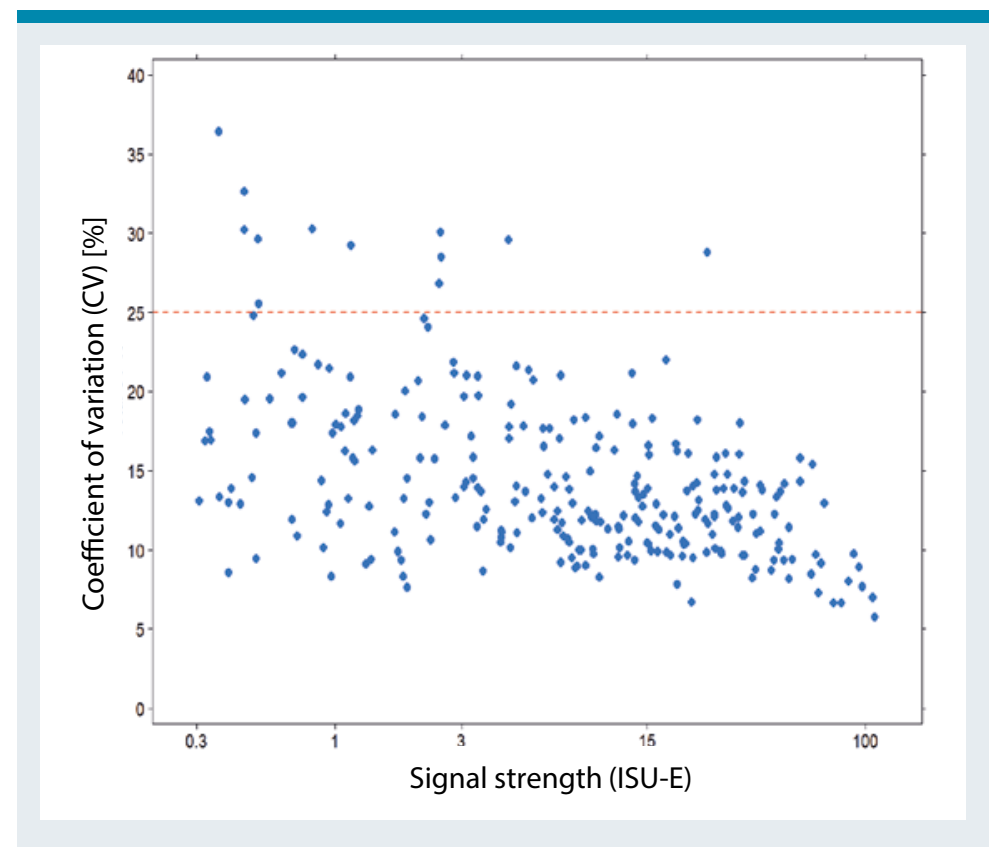

Fig. 2: Coefficient of variation (CV) depending on signal strength (ISU-E). Four serum samples covering 105 single allergens were used for calculation. Each sample was analyzed in triplicate in a total of 17 runs over a 4-week period. (From [8], with kind permission from Thermo Fisher Scientific, Uppsala, Sweden)

Tab. 1: Representative examples of coefficients of variation for slgE measurements against single allergens depending on signal strength

\begin{tabular}{|l|l|l|l|l|l|}
\hline Sample & Allergen & $\begin{array}{l}\text { Signal } \\
\text { strength ISU-E }\end{array}$ & $\begin{array}{l}\text { Mean } \\
\text { ISU-E }\end{array}$ & $\begin{array}{l}\text { CV intra-assay } \\
\text { variance (\%) }\end{array}$ & $\begin{array}{l}\text { CV inter-assay } \\
\text { variance (\%) }\end{array}$ \\
\hline 1 & rPar j 2 & $0.33-0.98$ & 0.32 & 18 & 9 \\
\hline 2 & nGal d 1 & & 0.46 & 11 & 16 \\
\hline 3 & nCry j 1 & & 0.98 & 12 & 13 \\
\hline 4 & rEqu c 1 & $1.2-14$ & 1.2 & 15 & 11 \\
\hline 5 & rDer f 1 & & 4.6 & 5 & 9 \\
\hline 6 & rFel d 1 & & 14 & 8 & 9 \\
\hline 7 & rAra h 1 & $19-90$ & 19 & 11 & 13 \\
\hline 8 & rPhl p 5b & 47 & 6 & 7 \\
\hline 9 & rBet v 1 & 90 & 7 & 7 \\
\hline$C V$, coefficient of variation & & &
\end{tabular}

Tab. 2: Averaged coefficient of variation for all allergens depending on signal strength

\begin{tabular}{|l|l|l|l|}
\hline ISU-E & Class & CV intra-assay variance (\%) & CV inter-assay variance (\%) \\
\hline $0.3-1$ & Low & 7 & 14 \\
\hline $1-15$ & Moderate & 6 & 10 \\
$>15$ & High & 5 & 9 \\
\hline
\end{tabular}



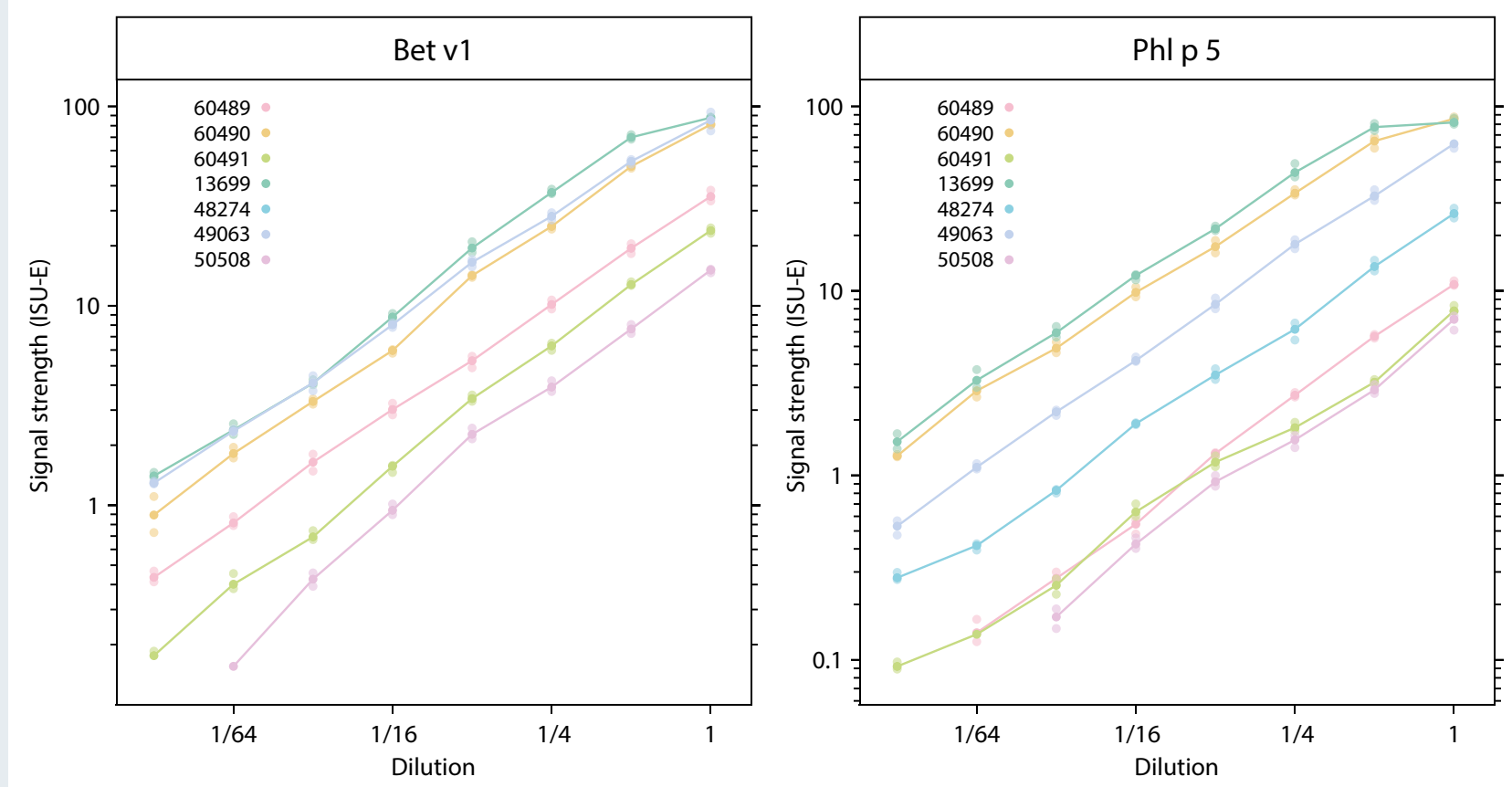

Fig. 3: Linearity of measurements in a dilution series using the examples of Bet $v 1$ and Phl p 5: serial 1:2 dilutions of seven different sera with slgE values > 5 ISU-E/I. (From [8], with kind permission from Thermo Fisher Scientific, Uppsala, Sweden)

Sample material and interference: Investigations comparing sample materials were carried out on serum, citrate, heparin, and ethylenediaminetetraacetic acid (EDTA) plasma from identical

Tab. 3: Representative data on linearity (slope) and the coefficient of determination $\left(R^{2}\right)$ of different allergens

\begin{tabular}{|l|l|l|}
\hline Allergen & Slope & $\mathbf{R}^{2}$ \\
\hline rAra h 2 & 1.03 & 0.96 \\
\hline rBer e 1 & 1.07 & 0.97 \\
\hline rBet v 1 & 1.16 & 0.95 \\
\hline rCan f 1 & 1.12 & 0.92 \\
\hline nCyn d 1 & 1.09 & 0.91 \\
\hline rDer f 2 & 1.01 & 0.99 \\
\hline rEqu c 1 & 1.18 & 0.93 \\
\hline nGal d 1 & 1.01 & 0.99 \\
\hline nPen m 1 & 1.07 & 0.97 \\
\hline rPhl p 1 & 1.12 & 0.97 \\
\hline
\end{tabular}

donors. These comparisons showed that serum, citrate, or heparin plasma from capillary or venous blood can be used. Using EDTA plasma can cause interference with $\mathrm{Ca}^{++}$-binding allergens (e.g., Gad c 1 , Pen $\mathrm{m} \mathrm{4}$, or polcalcin Bet v 4 and Phl p 7) and thus lead to false-negative or false-low results. When testing hemolytic or lipemic samples, neither hemolysis (up to $5 \%$ ) nor hypertriglyceridemia (triglyceride concentration up to $12 \mathrm{mg} / \mathrm{ml}$ ) caused significant interference in the test system.

A factor known to influence the determination of sIgE in solid-phase assays is the level of total IgE. In order to test this influence, an IgE-negative serum sample and four serum samples exhibiting sIgE to 68 of the 112 allergens were spiked with high total-IgE concentrations (3000 or $10,000 \mathrm{kU} / \mathrm{l})$ and measured simultaneously. As shown in Fig. 4, supplementing high concentrations of total IgE had no effect on test performance.

\section{Comparison of sigE to single allergens determined in multiplex (ISAC sIgE 112) and singleplex assays (ImmunoCAP)}

Using 350 sera and 57 allergens that were also available as ImmunoCAP singleplex reagents, the manufacturer compared the two different assay systems. Depending on the frequency of sensitization, a 
correlation of the measured values was demonstrated for each allergen with at least five, maximally 75 sera. As shown by way of example in Fig. 5, a good to very good correlation of the ISU-E values with the ImmunoCAP-derived values $\left(\mathrm{kU}_{\mathrm{A}} / \mathrm{l}\right)$ was observed for many allergens. However, the test sensitivity of ImmunoCAP is clearly higher for some allergens (i.e., LoD is lower). Another investigation used sera from 82 patients and a total of 555 measurements of sIgE to single allergens to compare the two methods [9]. Using negative cutoff values of $<0.3$ ISU-E and $<0.35 \mathrm{kU}_{\mathrm{A}} / \mathrm{l}\left(\right.$ or $\left.<0.1 \mathrm{kU}_{\mathrm{A}} / \mathrm{l}\right)$, a concordance rate of $92.2 \%$ (or $78.7 \%$ ) was found for the positive results. The concordance rate for the negative findings was $93.6 \%$.

Although excellent concordance rates were seen for most allergens, clear discrepancies were shown for isolated allergens. These included rAsp f $1(9 / 14)$, rPru p 3 (5/13), nAna c 2 (4/11), and rApi g 1 (4/10) [9]. Differences in the performance of individual allergens can potentially be explained by the differing presentation of allergens on the solid-phase of the assay. Compared with immobilization on the polymer coating of the glass chips, covalent binding of allergens to the cellulose matrix in the CAP system can result in different epitopes being exposed or blocked, and thus to suboptimal binding of sIgE present in the sample. Additional differences be-

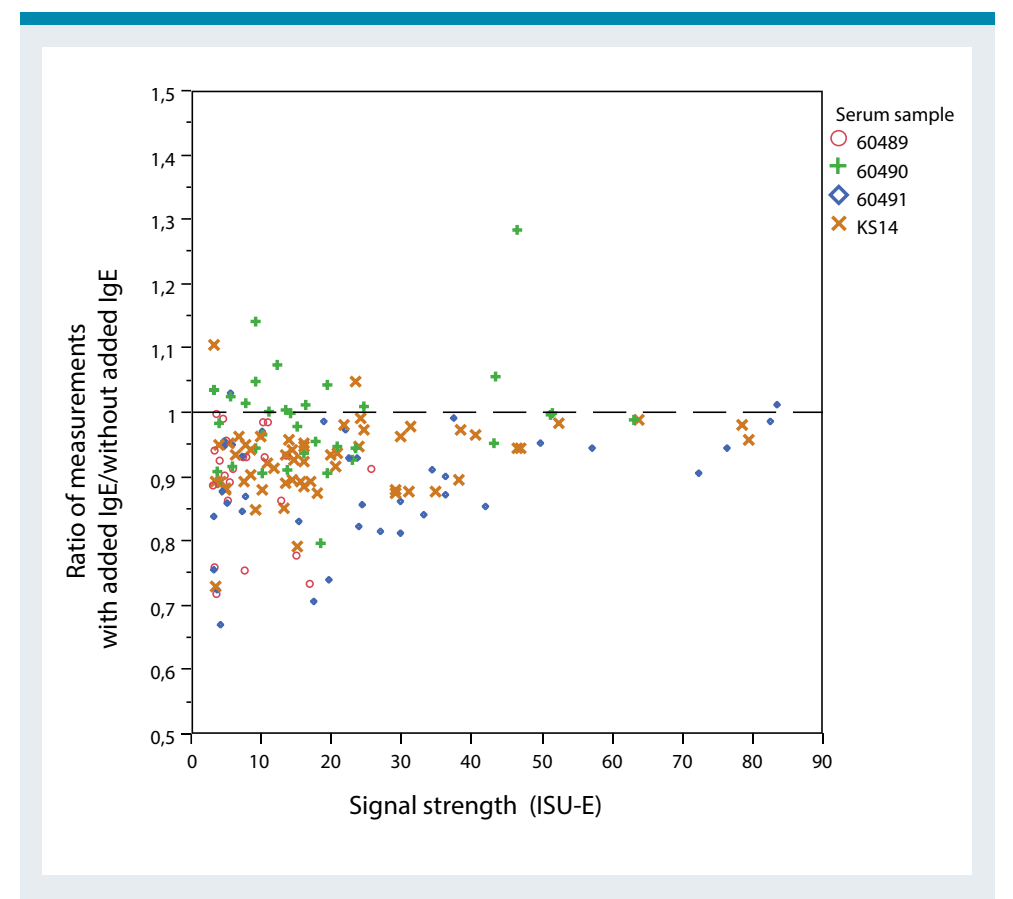

Fig. 4: The addition of high concentrations of IgE has no significant effect on measurements. The ratios of the measurement values obtained with and without the addition of $\lg E(10,000 \mathrm{kU} / \mathrm{l})$, as measured in different serum samples, are shown. (From [8], with kind permission from Thermo Fisher Scientific, Uppsala, Sweden)
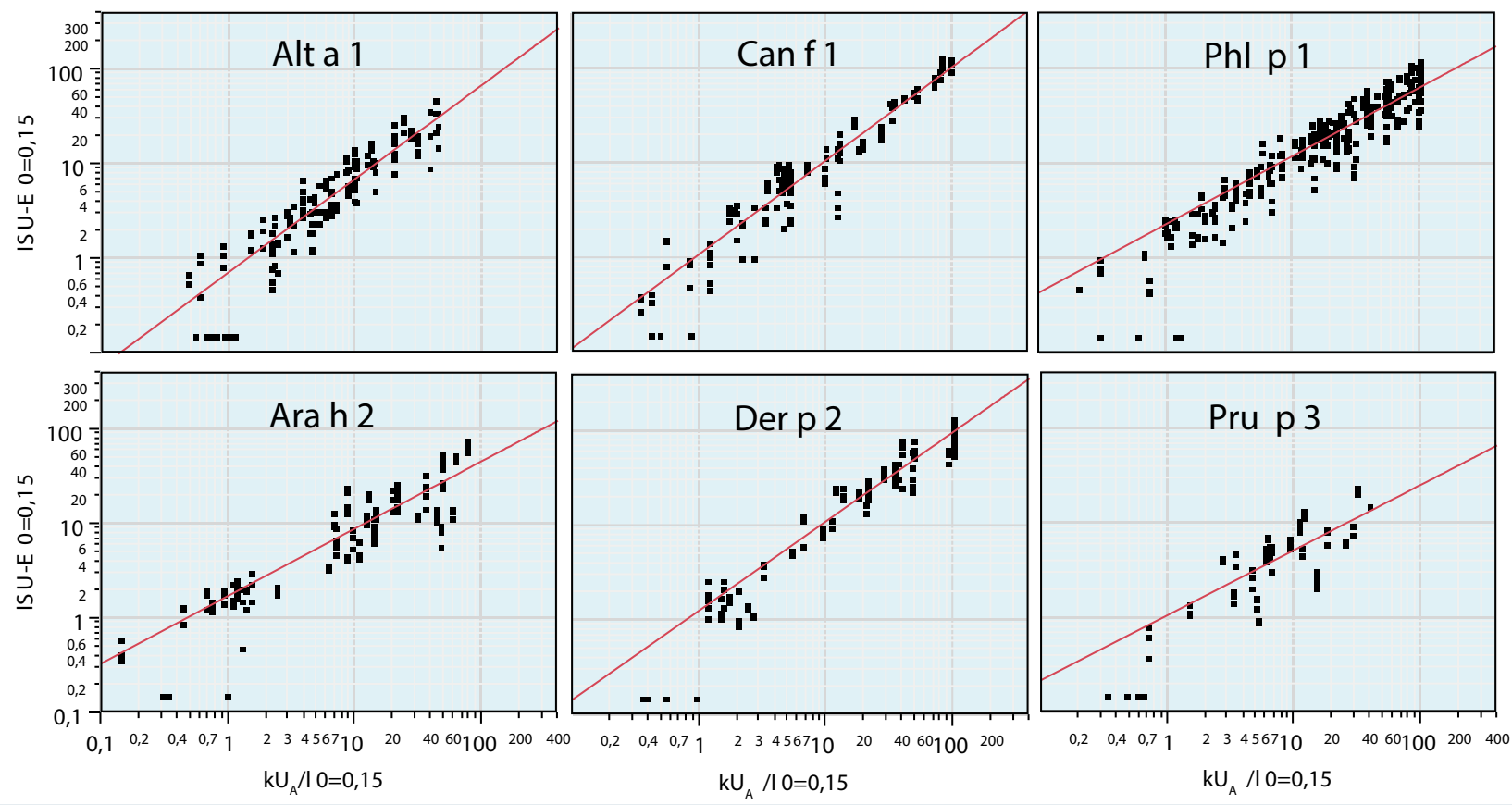

Fig. 5: Comparison of measurements made using the ISAC slgE 112 (ISU-E) and ImmunoCAP $\left(\mathrm{kU}_{\mathrm{A}} / \mathrm{l}\right)$ systems for selected single allergens. Negative results $(<0.15)$ are plotted as $0.15 \mathrm{ISU}-\mathrm{E}$ or $\mathrm{kU}_{\mathrm{A}} / \mathrm{l}$. (From [8], with kind permission from Thermo Fisher Scientific, Uppsala, Sweden) 
tween the setups of the two test systems can cause discrepant results in particular cases. Whereas a large excess of allergen is present in the ImmunoCAP system, thus leading to binding of all sIgE present in the sample in most cases, approximately $10^{6}$-fold less allergen is present in the ISAC assay. This can mean that not all allergen-specific IgE will find a binding partner, thus leading to lower results. In this respect, other allergen-specific antibody isotypes (particularly IgG) play a significant role, since these can also block the IgE binding sites (IgE epitopes), resulting in false-low IgE concentrations. On the other hand, the kinetics generated by the large excess of allergen in the ImmunoCAP singleplex assay allow binding of low-affinity sIgE, whereas the kinetics of ISAC 112 ensure that high-affinity sIgE is preferentially bound.

\section{Molecular allergy diagnostics using multiplex assays in clinical routine Allergen spectrum available and potential advantages in diagnostics}

With 112 individual allergens from 51 allergen sources, the ImmunoCAP ${ }^{\circledast}$ ISAC 112 assay currently offers the widest allergen spectrum for molecular allergy diagnostics in clinical routine. Particularly those allergens were selected that:

- frequently cause sensitizations and/or

_ confer an additional benefit in the interpretation of individual sensitization profiles.

The current version of the allergen chip includes: -43 single allergens from 17 different foods

-30 single allergens from 16 different seasonal aeroallergen sources

-27 single allergens from 13 different perennial aeroallergen sources

- 12 additional single allergens from other allergen sources

Detailed analysis of IgE sensitizations using the allergen chip enables differentiated diagnostics, whereby the advantages of broad molecular screening is evident, even without knowledge of clinical symptoms, from a universal analytical perspective (on the test level). The following consequences or particular arguments should be considered when using these single allergens in microarray format:

A. Increased test sensitivity (low limit of quantification, LoQ) achieved by using specific single allergens compared with diagnostics using allergen extracts

B. Improved analytical specificity (selectivity) for particular single allergens with special characteristics (e.g., IgE sensitization associated with severe reactions)

C. Indicators of cross-reactivity (common cause of a lack of analytical specificity of allergen extracts)
D. Markers of primary, genuine (possibly species-specific) IgE sensitization

E. Ideally, complete representation of the individual sensitization profile (in contrast to singleplex specific molecular IgE diagnostics)

Criterion A: Individual allergens underrepresented or lacking in an allergen extract can bind sIgE better when used in a targeted manner in the microarray, thus generating positive signals and indicating sensitizations more accurately. However, the LoQ is usually lower for singleplex methods than it is for microarrays, due to the large amounts of (single) allergen used. This explains the limited precision and accuracy of microarrays at sIgE concentrations below $1 \mathrm{kU}_{\mathrm{A}} / \mathrm{l}$. Therefore, especially sera with low total $\operatorname{IgE}(<25 \mathrm{kU} / \mathrm{l})$ can yield false-negative values to certain single allergens in the microarray analysis; therefore singleplex testing is preferred (to microarray) in such constellations.

Criterion B: Increased analytical specificity is especially desirable when the specific physicochemical characteristics of the single allergens concerned are associated with particular clinical consequences (e.g. high allergen stability and/or high proportion of the total allergen source as the cause of risk-associated sensitizations, e.g. to particular foods; localization of the allergens as a means of differentiating between certain clinical presentations, e.g. sIgE to intracellular Aspergillus allergens in bronchopulmonary aspergillosis).

Increased analytical specificity is not an advantage per se: only when the selective information regarding the allergen in an extract is associated with a predefined (clinical) characteristic does this have a significant benefit for molecular diagnostics.

Criterion C: Single allergens improve, in particular, the allergen specificity of IgE sensitization tests. In light of this, certain conserved allergen molecules that:

_are of similar structure

_ have common IgE-binding epitopes

_occur in numerous allergen sources

have proven useful as indicators for identifying potential cross-reactivity. They form the basis for concomitant sensitizations to different allergen sources with quite variable biological relationships.

Criterion D: Other single allergens, in contrast, yield important information regarding a genuine primary IgE sensitization on the basis of:

_ their well-defined, particular structure

_ their IgE epitopes with limited similarity in other single allergens

- their presence in highly specific allergen sources. 
Single allergens re-establish the necessary analytical specificity, particularly in the case of allergen sources with known cross-reactive single allergens.

Criteria A-D are by no means mutually exclusive, since single allergens can embody several advantages. Their value in molecular diagnostics (in both singleplex and multiplex assays) varies for each allergen molecule from case to case, and must be redefined based on the specific question.

Criterion E: In contrast to singleplex testing, multiplex assays ideally reveal all potential sensitizations. This discloses the entire spectrum of an individual's susceptibility to allergy, and the allergen-specific IgE repertoire can then be systematically checked for present or absent clinical relevance. This procedure is currently also referred to as a bottom-up approach (in contrast to the topdown approach based on medical history, extract based tests, followed by specific singleplex testing using single allergens).

\section{Additional benefit conferred by molecular allergy diagnostics in clinical routine \\ Differentiation between genuine sensitization and cross-reactivity with inhalant allergens: In pollen allergy patients exhibiting serological or skin test reactivity to various pollen species (e.g., birch, grasses, mugwort), this may indicate either a genu- ine sensitization to the particular type of pollen or be caused by IgE cross-reactivity to cross-reactive panallergens, such as, e.g., \\ - profilins (e.g., Bet v 2, Phl p 12, Art v 4, Amb a 8) \\ _polcalcins (e.g., Bet v 4, Phl p 7, Art v 5, Amb a 10)}

Differentiation between a genuine sensitization and cross-reactivity is only possible if IgE reactivity to specific marker allergens can be demonstrated. Only then does the reactivity result from a genuine primary sensitization to the relevant allergen source. To enable such a distinction to be made, the ISAC 112 assay features numerous marker allergens from different pollen species, including:

_ Bet v 1 for birch pollen

_Ole e 1 for ash pollen

_ Pla a 1 for plane pollen

- Cup a 1 for cypress pollen

_ Phl p 1, Phl p 2, Phl p 5, Phl p 6, Phl p 11 for grass pollen

—Art v 1 for mugwort pollen

-Amb a 1 for ragweed

_Pla 11 for buckhorn plantains (e.g. Plantago lanceolata)

- Che a 1 for goosefoot

At the same time, the IgE reactivity to panallergens such as
- profilins (Phl p 12, Bet v 2)

- polcalcins (Phl p 7, Bet v 4)

can be determined in order to obtain information on potential cross-reactivity. To what extent panallergens can contribute to allergic reactions and clinical manifestations of pollen allergies is still the subject of debate. However, due to their high degree of cross-reactivity, these panallergens represent a considerable problem for the detection of allergen-specific sensitization using extract-based methods. For this reason, it is particularly important to perform sIgE diagnostics using species-specific marker allergens in polysensitized patients, alongside a consideration of the precise medical history. These tests yield information relevant to selecting the correct extract prior to commencing immunotherapy. Diagnostic testing using the ISAC 112 multiplex platform reveals an extensive sensitization profile, including the most common markers and cross-reactive allergens, in a single measurement.

Identification of sensitizations to food allergens associated with a high risk for severe allergic reactions: IgE to food extracts can be the result of cross-reactivity with pollen-associated allergens, such as, e. g., allergens of the Bet $\mathrm{v} 1$ or profilin families.

Pollen allergens of the Bet $\mathrm{v} 1$ family include:

- Bet v 1 (birch)

- Aln g 1 (alder)

- Cor a 1 (hazel)

- Que a 1 (oak)

_Fag s 1 (beech)

In the case of relevant sensitization to these aeroallergens, cross-reactivity with the following food allergens is common due to high sequence and structural homology:

- pome and stone fruits, nuts (hard-shelled fruits), e.g.: Act d 8 (kiwi), Cas s 1 (chestnut), Cor a 1 (hazel), Fra a 1 (strawberry), Mald 1 (apple), Pru p 1 (peach), and Pyr c 1 (pear) _ vegetables and legumes, e.g.: Api g 1 (celery), Ara h 8 (peanut), Dau c 1 (carrot), Gly m 4 (soy), and Vig $r 1$ (mung bean)

Similarly, it is assumed that sensitization to pollen-mediated profilins can cause cross-reactivity with corresponding profilins in food. The pollen profilins responsible for sensitizations in regions with high grass pollen counts are mainly grass pollen profilins, such as Phl p 12 (timothy grass). Less frequently, Bet v 2 (birch) or Art v 4 (mugwort) - in other regions possibly Amb a 8 (ragweed) or Ole e 2 (olive) - can also cause profilin sensitization.

In terms of food, corresponding profilins are present in fruits, e.g.:

_Ana c 1 (pineapple)

- Cit s 1 (orange) 
-Cuc m 2 (melon)

_-Fra a 4 (strawberry)

_Mal d 4 (apple)

as well as in legumes and vegetables:

- Ara h 5 (peanut)

_ Gly m 3 (soy)

-Api g 4 (celery)

-Cap a 2 (bell pepper)

_Dau c 4 (carrot)

_Lyc e 1 (tomato)

The Bet $\mathrm{v} 1$ and profilin family allergens are sensitive to heat and digestion, and generally only cause local oropharyngeal symptoms. Exceptions to this may be observed if large quantities of untreated, "native" allergens are consumed. In the absence of heat treatment or previous processing and denaturation of proteins, systemic reactions may occur. A classic example of this is consumption of native soy milk by individuals highly sensitized to Gly $\mathrm{m} 4$.

In contrast to pollen-associated food allergies to Bet v 1 homologs or profilins, sensitization to food allergens from the storage protein families are frequently associated with a significantly increased risk for severe allergic reactions: storage proteins are extremely resistant to heat and digestion, and are present in legumes and tree nuts in large quantities. A distinction is made between different storage protein families:

_11S globulins (legumins),

—7S globulins (vicilins)

_2S albumins

The following nut storage proteins are characterized:

— hazelnut: Cor a 9, Cor a 11, Cor a 14

_walnut: Jug r 1, Jug $r 2$ and Jug r 4

-pecan nut: Car i 1, Car i 2, Car i 4

_almond: Pru du 6

_cashew: Ana o 1, Ana o 2, Ana o 3

- pistachio: Pis v 1 , Pis v 2 , Pis v 3 , Pis v 5

_brazil nut: Ber e 1, Ber e 2

Among the legumes:

_peanut: Ara h 1, Ara h 2, Ara h 3, Ara h 6

_ soy: Gly $\mathrm{m} 5$, Gly $\mathrm{m}$ 6, and Gly $\mathrm{m} 8$

The detection of sIgE to specific storage proteins serves as indicator for an increased risk of severe allergic reactions to small quantities of the allergen. IgE detections to the following allergens are particularly important:

- Ara h 2 in peanut allergy

_ Cor a 9 and Cor a 14 in hazelnut allergy

_ Jug r 1 and Jug r 4 in walnut allergy

— Ber e 1 for brazil nuts

Similarly, the detection of sIgE to members of the lipid transfer protein (LTP) family appears to be associated with an increased risk of systemic reactions. This includes peach LTP Pru p 3 - particularly in patients from Mediterranean regions, who have been sensitized cutaneously by the high LTP content of the skin of ripe peaches - as well as walnut Jug r 3 and hazelnut Cor a 8. Since many of the aforementioned allergens are present on the allergen chip, the ISAC 112 multiplex diagnostic test largely reveals individual sensitization profiles and thus forms the basis for risk assessment during subsequent patient counseling.

Interpretation supported by intelligent software, results evaluated by the physician

Using ISAC 112 to simultaneously determine 112 parameters in order to generate detailed sensitization profiles presents a challenge for the physician, particularly in the case of polysensitized patients. The manufacturer's X-plain software integrated into the ISAC 112 system ensures a systematic compilation of positive results and simplifies interpretation of the relevance of the detected sensitizations.

The X-plain software can of course only deliver background information on the different allergens, and the results of the extensive sensitization test are not a substitute for an expert medical diagnosis. Therefore, all reports on diagnostic findings include a corresponding statement that the detection of $\operatorname{IgE}$ must always be evaluated in combination with the clinical medical history, and that the computer-generated information is intended to assist the treating physician in making a clinical diagnosis and not to replace him/her.

In addition to the X-plain software developed by the manufacturer as an aid to interpretation, the "Allergenius ${ }^{\oplus "}$ software-based expert system, which supports the interpretation of ISAC data according to similar principles, has also been recently introduced [10]. In addition to ISAC data, data from skin prick tests and individual sIgE determinations can also be entered in the Allergenius ${ }^{\circledast}$ system and included in the computer-generated report. It can be assumed that expert systems such as X-plain or $\mathrm{Al}$ lergenius ${ }^{\otimes}$ will develop rapidly and further simplify the interpretation of complex sensitization profiles in the future.

\section{Special features in routine use}

Own experience with the test system in routine diagnostics performed at a large outpatient allergy clinic have shown that positive sIgE values are rarely measured using ISAC 112 when total-IgE concentrations are below $25 \mathrm{kU} / \mathrm{l}$. Therefore, the test is now generally only performed when the total-IgE concentration exceeds $25 \mathrm{kU} / \mathrm{l}$.

Of the 112 allergens, six are glycosylated, i.e., have carbohydrate side chains that can bind IgE. These include walnut nJug r 2, Bermuda grass nCyn d 1, Timothy grass nPhl p 4, Japanese cedar nCry j 1, Arizona cypress nCup a 1 , and plane nPla a 2 . Since it 
is not possible to determine whether IgE to these six allergen components is directed to the protein part or the carbohydrate side chain, the results need to be evaluated with caution and in the context of $\operatorname{IgE}$ reactivity to the CCD marker MUXF3.

\section{Molecular allergy diagnostics using multiplex assays in research New insights gained using ISAC technology}

The small sample volumes required for multiplex assays are advantageous in the research environment, e.g., in the context of birth cohorts, since only small amounts of serum are normally available for analysis. These options made it possible to collect, e.g., the following data:

Diversity of sensitization profiles: By means of simultaneous determination of sIgE antibodies to numerous allergen molecules, patients' individual sensitization profiles can be generated with minimal effort. These profiles represent the IgE repertoire and pattern of sensitization at the molecular level and enable the great diversity of profiles in a population to be depicted. Tripodi et al. [11] alone described 39 different profiles (sensitization patterns) in only 176 Italian, grass pollen-allergic children that were tested using eight Phleum pratense (Timothy grass, Phl p) allergens: the spectrum extended from children who reacted to only one molecule, to children who produced antibodies to all eight allergens. A range of intermediate profiles exists between these two extremes.

Developing sensitization profiles: It could be shown using the ISAC method that sensitization profiles of children are simple to begin with and in crease in complexity over time: the sIgE response to the Phleum pratense allergen molecules often develops from a simple monosensitization to a single allergen molecule into an oligomolecular sensitization, leading ultimately to a complex polymolecular pattern $[12,13]$. This development process usually begins with an IgE response to a starter molecule, which, in later stages, initiates the development of antibodies to other allergen molecules. In the case of grass pollen allergy to timothy, this starter molecule is usually $\mathrm{Phl} \mathrm{p} \mathrm{1}$, which turned out to be the protein most frequently recognized. As a result, young patients in the early stages of their sensitization often exhibit a sIgE response to only this protein. After months or years, IgE sensitizations to other Timothy grass proteins can develop, commonly in a typical order: the initial sensitization to $\mathrm{Phl} \mathrm{p} 1$ is usually followed by positive reactions to $\mathrm{Phl}$ p 4 and Phl p 5; thereafter IgE responses to Phl p 2, Phl p 6, and Phl p 11. Only in the clinical phase, long after all allergic symptoms had devel- oped in these children, was it possible to detect IgE to $\mathrm{Phl}$ p 12 and Phl p 7 - pollen panallergens with a low risk of sensitization. The time-dependent, consecutive development of allergen molecule-specific IgE sensitizations to an allergen source (grass pollen in this example) is described by the authors as "molecular spreading" [12].

Since the first sIgE responses to pollen are detectable years before the first symptoms occur, ISAC microarray analysis might be able to predict symptom onset on the basis of the individual sensitization profile. Indeed, approximately two third of 3 -year-old children sensitized to grass pollen develop grass pollen-associated seasonal rhinitis at the age of 12 years [12]. Similar results were recently reported for the development of birch pollen-associated allergic rhinoconjunctivitis [14]. Here again, IgE reactivity to various Bet v 1-homologous PR-10 proteins in early childhood seems to be a good predictor for the later development of a clinically manifest birch pollen allergy.

Prescribing behavior in allergen-specific immunotherapy (SIT): Recommendations on SIT also take into consideration the efficacy of this therapy depending on how well it is adapted to the allergen sources to which the patient reacts [15]. SIT should be used in the case of clinical symptoms arising from IgE sensitizations to clearly definable allergen sources, including their primary major allergens, without taking cross-reactivity toward panallergens of questionable clinical relevance into consideration [16]. The multiplex ISAC 112 system generates differentiated sensitization profiles, thus enabling "primary“ genuine sensitizations to be distinguished from antibody reactions resulting from cross-reactivity. The advantage here is that it could allow SIT to be individually tailored to each patient. Thus, current German guidelines on SIT [17] recommend diagnostics using specific single allergens in polysensitized pollen allergy patients - preferentially in singleplex rather than multiplex procedures - since generating complete sensitization profiles to more than 100 allergens just to answer the specific diagnostic questions of pollen sensitization would in many cases be unnecessary diagnostic excess.

A multicenter Italian study [18] has already investigated whether and how the results of molecular allergy diagnostics using singleplex assays influence physicians' prescription of SIT and decisions relating to the composition of allergen preparations for children with moderate to severe allergic rhinitis $(\mathrm{n}=651)$. This study revealed that more SIT preparations were prescribed following molecular diagnostics: in many patients originally classified as polysensitized on the basis of skin prick 
tests with pollen extracts, molecular diagnostics could identify clear sensitizations to particular major allergens, the allergen sources of which would then have come into consideration for SIT. The detection of IgE to primary major allergens thus re-establishes the analytical specificity that was lost by using allergen extracts for diagnostic purposes due to pan-pollen sensitizations. In addition, it could be shown that, in approximately $33 \%$ of cases, SIT would have been adjusted and performed with a different composition following molecular diagnostics.

\section{The use of individually tailored allergen chips in research}

In addition to the test systems approved for $\operatorname{sigE}$ routine diagnostics (e.g., ImmunoCAP ${ }^{\circledR}$ ISAC 112 sIgE), protein microarrays can also be developed to address specific research questions. On the basis of ISAC technology, a significantly more extensive allergen chip was developed - e.g., for birth cohort-based investigations on the mechanisms of allergy development in different regions of Europe - on which a total of 176 allergen components are represented [19]. In a similar manner, individually designed protein microarrays can be used as allergen chips in order to answer specific research questions. Thus, customized microarrays were able to detect sIgE to various chimeric isoforms of Api m 10, a major allergen in honey bee venom [20]. The roles of sIgE to $\alpha$-, $\beta$-, or $\gamma$-gliadin in wheat-dependent exercise-induced anaphylaxis were also characterized using research microarrays [21], as was the rel-

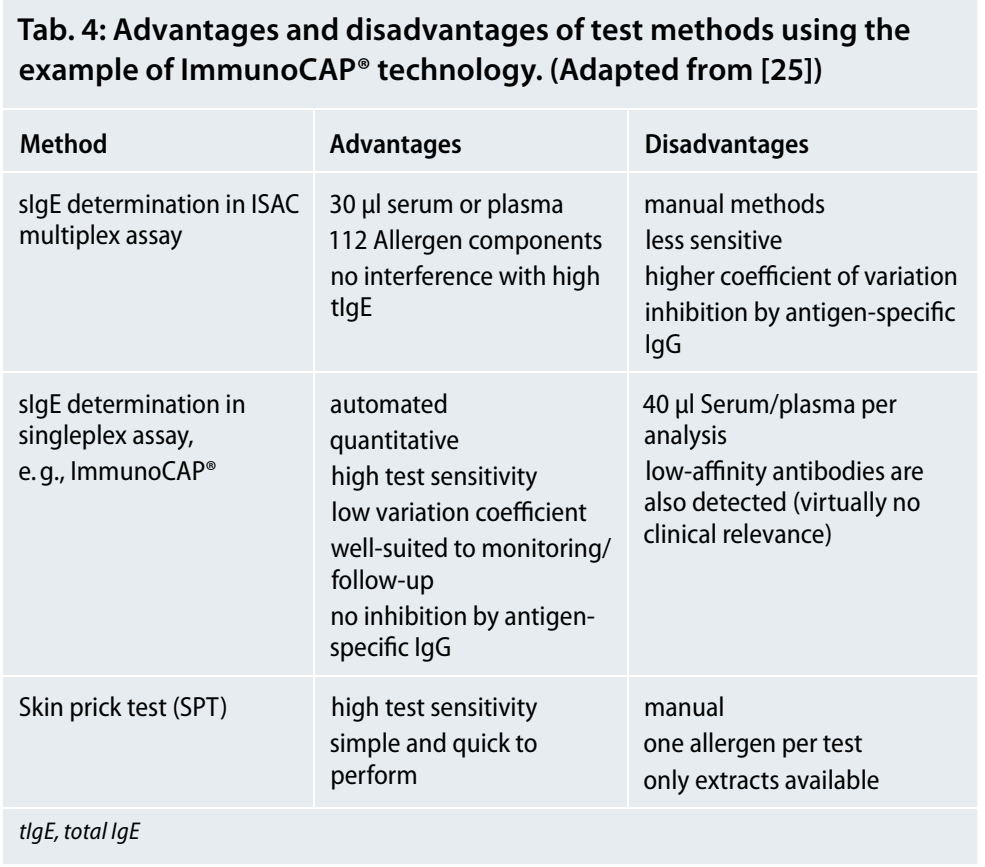

evance of the different single allergens for peanut allergy [22].

A further application of array technology can be illustrated using the example of peanut allergy: rather than intact proteins, allergen peptides can also be coupled to the solid phase of the array as target structures. This type of peptide array permits the analysis of diverse linear IgE binding sites (IgE epitopes) within an allergen [23] and their comparison with homologous sequences in other allergens [24].

The clear advantages of the multiplex assay for research purposes lie in the large number of detectable sensitizations, the individual composition of the allergen repertoire (personalized allergen chips), and the relatively small sample volumes required for the actual test. Particularly in the case of complex allergen sources and complicated clinical questions, or in a polysensitized study population, high-definition molecular allergy diagnostics are beneficial, since the complete sensitization pattern obtained is a prerequisite for the successful interpretation of results in the context of the patient's clinical medical history.

\section{Conclusion and future directions}

The ISAC 112 microarray platform currently available enables the analysis of specific IgE to as many single allergens as possible in a single assay, using a small amount of serum (Tab. 4). Strictly speaking, the assay represents 112 immunoassays, the corresponding allergen components of which are derived from natural or recombinant sources and have been individually evaluated for their suitability. This relates to allergen-dependent test parameters, such as LoD, linearity, precision, effect of total IgE, IgG inhibition, matrix effects, and comparability with established methods for detecting specific IgE to defined single allergens.

The analytical advantages of molecular diagnostics using single allergens also apply to multiplex analysis:

_ increased test sensitivity (lower LoD) by using specific (e.g., allergens under-represented or lacking in the allergen extract) single allergens

_increased analytical specificity (selectivity) for single allergens with defined clinical characteristics (e.g., risk association, disease association) _ defined single allergens (e.g., panallergens) as indicators of cross-reactivity

— single allergens (e.g., species-specific major allergens) as markers for a primary, genuine IgE sensitization to the associated allergen source.

The additional advantage of multiplex analysis is that it generates an extensive (ideally complete) IgE sensitization profile (complete allergen-specific IgE repertoire). 
Since the reliability and accuracy of the current microarray test decreases significantly at sIgE concentrations below $1 \mathrm{kU} / \mathrm{l}$, singleplex methods are where possible - to be preferred over multiplex assays in the case of low serum total $\operatorname{IgE}(<25 \mathrm{kU} / \mathrm{l})$ or only slightly increased sIgE values $(0.1<\operatorname{sIgE}<1.0$ $\left.\mathrm{kU}_{\mathrm{A}} / \mathrm{l}\right)$.

A number of important allergen components, particularly in the area of food allergens (e.g., additional storage proteins; potentially important pollen allergens; mould allergens; animal allergens) are lacking. Other allergen components currently featured on the allergen chip would be better dispensed than included, since they lead more to confusion than to clarification. These include insect venom allergens, since analysis of specific IgE to these allergens is only indicated on the basis of clear signs of an anaphylactic reaction to insect stings in the patient's medical history, and not as a screening test. Due to the high prevalence of insect venom sensitization in approximately $25 \%$ of the population, nonspecific screening would generate an abundance of clinically irrelevant results and serve to unsettle patients and their physicians. On the basis of the appropriate indication, sensitization to single insect venom allergens can be detected using singleplex methods. Alternatively, specific multiplex analysis with all available insect venom allergens a so called insect venom allergen chip that is currently under development - would be useful. In this regard, it is conceivable that a range of microarray formats will be available in the future, which, depending on the clinical question, will cover different allergen spectra, such as for example food allergies, inhalant allergies, insect venom allergies, and drug allergies. In light of the fact that there are probably over 3000 single allergens, it can be expected that the rapid developments in miniaturization and automation will fuel many more innovations in the field of multiplex allergy diagnostics.

\section{Prof. Dr. Thilo Jakob}

Department of Dermatology and Allergology University Medical Center Gießen and Marburg Justus Liebig University Gießen

Gaffkystrasse 14

35385 Gießen, Germany

E-Mail: thilo.jakob@derma.med.uni-giessen.de

\section{Conflict of interest}

TJ received research funds from Phadia/Thermo Fisher Scientific, Freiburg, Germany, and Dr. Fooke Laboratories $\mathrm{GmbH}$, Neuss, Germany, as well as honoraria for lectures and advisory activities from Phadia/Thermo Fisher Scientific. PF is employee of Phadia/Thermo Fisher Scientific, Vienna, Austria. PM has received research support and honorarium for lectures from Phadia/ThermoFisher $\mathrm{GmbH}$, Freiburg, Germany, Euroimmun GmbH, Lübeck,
Germany and HYCOR BioMedical, Indianapolis, IN, USA. JKT has received research support and honoraria for lectures from Dr. Fooke GmbH, Neus, Germany and Phadia/ ThermoFisher GmbH, Freiburg, Germany.

\section{Cite this as}

Jakob T, Forstenlechner P, Matricardi P, Kleine-Tebbe J. Molecular allergy diagnostics using multiplex assays: methodological and practical considerations for use in research and clinical routine. Part 21 of the Series Molecular Allergology. Allergo J Int 2015;24:320-32

DOI: 10.1007/s40629-015-0056-2

\section{References}

1. Blackley CH. Hay fever; its causes, treatment, and effective prevention. London: Ballière; 1880

2. Ishizaka K, Ishizaka T. Identification of gamma-E antibodies as a carrier of reaginic antibody. J Immunol 1967;99:1187-98

3. Johansson SGO, Bennich $\mathrm{H}$. Immunological studies of an atypical (myeloma) immunoglobulin. Immunology 1967;13:381-94

4. Wide L, Bennich $H$, Johansson SG. Diagnosis of allergy by an in vitro test for allergen antibodies. Lancet 1967;2:1105-7

5. Breiteneder $\mathrm{H}$, Hassfeld W, Pettenburger K, Jarolim E, Breitenbach M, Rumpold $\mathrm{H}$ et al. Isolation and characterization of messenger RNA from male inflorescences and pollen of the white birch (Betula verrucosa). Int Arch Allergy Appl Immunol 1988;87:19-24

6. Hiller R, Laffer S, Harwanegg C, Huber M, Schmidt WM, Twardosz A et al. Microarrayed allergen molecules: diagnostic gatekeepers for allergy treatment. FASEB J 2002:16:414-6

7. Kleine-Tebbe J, Jakob T. Molecular allergy diagnostics using IgE singleplex determinations: methodological and practical considerations for use in clinical routine. Allergo J Int. 2015; 24: 185-97

8. Thermo Fisher Scientific, ed. ImmunoCAP ${ }^{\circledR}$ ISAC 112 performance characteristics. Data on file, Oct 2011. Uppsala: Thermo Fisher Scientific; 2011

9. Gadisseur R, Chapelle JP, Cavalier E. A new tool in the field of in-vitro diagnosis of allergy: preliminary results in the comparison of ImmunoCAP 250 with the ImmunoCAP ISAC. Clin Chem Lab Med 2011;49:277-80

10. Melioli G, Spenser C, Reggiardo G, Passalacqua G, Compalati E, Rogkakou A et al. Allergenius, an expert system for the interpretation of allergen microarray results. World Allergy Organ J 2014;7:15

11. Tripodi S, Frediani T, Lucarelli S, Macrì F, Pingitore G, Di Rienzo Businco A et al. Molecular profiles of lgE to Phleum pratense in children with grass pollen allergy: implications for specific immunotherapy. J Allergy Clin Immunol 2012;129:834-9.e8

12. Hatzler L, Panetta V, Lau S, Wagner P, Bergmann RL, Illi S et al. Molecular spreading and predictive value of preclinical IgE response to Phleum pratense in children with hay fever. J Allergy Clin Immunol 2012;130: 894-901.e5

13. Matricardi PM. Allergen-specific immunoprophylaxis: toward secondary prevention of allergic rhinitis? Pediatr Allergy Immunol 2014;25:15-8

14. Westman $M$, Lupinek C, Bousquet J, Andersson N, Pahr S, Baar A et al; Mechanisms for the Development of Allergies (MeDALL) consortium. Early childhood IgE reactivity to pathogenesis-related class 10 proteins predicts allergic rhinitis in adolescence. J Allergy Clin Immunol 2015;135:1199-206.e1-11 
15. Zuberbier T, Bachert $C$, Bousquet PJ, Passalacqua G, Walter Canonica G, Merk H et al. GA ${ }^{2}$ LEN/EAACl pocket guide for allergen-specific immunotherapy for allergic rhinitis and asthma. Allergy 2010;65:1525-30

16. Valenta $R$. The future of antigen-specific immunotherapy of allergy. Nat Rev Immunol 2002;2:446-53

17. Pfaar O, Bachert C, Bufe A, Buhl R, Ebner C, Eng P et al. Guideline on allergen-specific immunotherapy in IgE-mediated allergic diseases: S2k Guideline of the German Society for Allergology and Clinical Immunology (DGAKI), the Society for Pediatric Allergy and Environmental Medicine (GPA), the Medical Association of German Allergologists (AeDA), the Austrian Society for Allergy and Immunology (ÖGAl), the Swiss Society for Allergy and Immunology (SGAl), the German Society of Dermatology (DDG), the German Society of Oto-Rhino-Laryngology, Head and Neck Surgery (DGHNO-KHC), the German Society of Pediatrics and Adolescent Medicine (DGKJ), the Society for Pediatric Pneumology (GPP), the German Respiratory Society (DGP), the German Association of ENT Surgeons (BV-HNO), the Professional Federation of Paediatricians and Youth Doctors (BVKJ), the Federal Association of Pulmonologists (BDP) and the German Dermatologists Association (BVDD). Allergo J Int 2014;23:282-319. DOI: 10.1007/s40629-014-0032-2

18. Stringari G, Tripodi S, Caffarelli C, Dondi A, Asero R, Di Rienzo Businco A et al; Italian Pediatric Allergy Network (I-PAN). The effect of component-resolved diagnosis on specific immunotherapy prescription in children with hay fever. J Allergy Clin Immunol 2014;134:75-81

19. Lupinek C, Wollmann E, Baar A, Banerjee $S$, Breiteneder $H$, Broecker BM et al. Advances in allergen-microarray tech- nology for diagnosis and monitoring of allergy: the MeDALL allergen-chip. Methods 2014;66:106-19

20. Vaerenbergh M van, De Smet L, Rafei-Shamsabadi D, Blank S, Spillner E, Ebo DG et al. IgE recognition of chimeric isoforms of the honeybee (Apis mellifera) venom allergen Api $\mathrm{m} 10$ evaluated by protein array technology. Mol Immunol 2015;63:449-55

21. Hofmann SC, Fischer J, Eriksson C, Bengtsson Gref O, Biedermann $T$, Jakob T. IgE detection to $\alpha / \beta / \gamma$-gliadin and its clinical relevance in wheat-dependent exercise-induced anaphylaxis. Allergy 2012;67:1457-60

22. Nicolaou N, Poorafshar M, Murray C, Simpson A, Winell H, Kerry $\mathrm{G}$ et al. Allergy or tolerance in children sensitized to peanut: prevalence and differentiation using component-resolved diagnostics. J Allergy Clin Immunol 2010;125:191-7.e1-13

23. Shreffler WG, Beyer K, Chu TH, Burks AW, Sampson HA. Microarray immunoassay: association of clinical history, in vitro IgE function, and heterogeneity of allergenic peanut epitopes. J Allergy Clin Immunol 2004;113: 776-82

24. Rosenfeld L, Shreffler W, Bardina L, Niggemann B, Wahn $\mathrm{U}$, Sampson HA, Beyer K. Walnut allergy in peanut-allergic patients: significance of sequential epitopes of walnut homologous to linear epitopes of Ara h 1,2 and 3 in relation to clinical reactivity. Int Arch Allergy Immunol 2012;157: 238-245

25. Canonica GW, Ansotegui IJ, Pawankar R, Schmid-Grendelmeier $P$, Hage $M$ van, Baena-Cagnani CE et al. A WAO ARIA - GA2LEN consensus document on molecular-based allergy diagnostics. World Allergy Organ J 2013;136:17-34 